Novon 11(1): 8-15. 2001

\title{
Passiflora tarminiana, a new cultivated species of Passiflora subgenus
}

\section{Tacsonia.}

Geo Coppens d'Eeckenbrugge ${ }^{1}$, Victoria E. Barney ${ }^{1}$, Peter Møller Jørgensen ${ }^{2}$, John M. MacDougal ${ }^{2}$

${ }^{1}$ CIRAD-FLHOR/IPGRI Project for Neotropical Fruits, c/o CIAT, A.A. 6713, Cali Colombia

${ }^{2}$ Missouri Botanical Garden, P.O. Box 299, St Louis, Missouri 63166-0299, U.S.A.

\begin{abstract}
The new species Passiflora tarminiana differs from its closes relative by the character combination of very small acicular stipules and large almost reflexed petals and sepals. This species has escaped detection despite being widely cultivated. Naturalized populations, particularly on Hawa'ii, have created problems for conservation of the native flora. In Colombia it is more frequently adopted in industrial cultivation because of its rusticity and resistance to fungal diseases.
\end{abstract}

\section{Introduction}

Passifloras of the subgenus Tacsonia are cultivated by many small farmers, from Venezuela to Bolivia. Some species are cultivated in New Zealand. The main cultivated species is Passiflora mollissima (Kunth) Bailey (Escobar, $1980 \&$ 1988), also called $P$. 
tripartita var. mollissima (Kunth) Holm-Nielsen \& P. Jørgensen (Holm-Nielsen et al., 1988). It is called "curuba de Castilla" in Colombia, "tacso de Castilla" in Ecuador, and "banana passionfruit" in English-speaking countries.. The second species of importance in the Andes is "curuba india," "curuba ecuatoriana," or "curuba quiteña" in Colombia, called "tacso amarillo" in Ecuador (Pérez Arbeláez, 1978; A.A.A., 1992; Campos, 1992). It is most frequently found in private gardens, but some commercial growers have, because of its rusticity, started to grow it instead of the "curuba de Castilla." We describe this overlooked cultigen as a new species under the name Passiflora tarminiana, in recognition of Tarmín Campos, a Colombian agronomist who contributed with enthusiasm to the development of banana passion fruit cultivation and introduced the first author to the cultivated passifloras of the central Colombian highlands.

Surprisingly, although producers and consumers easily differentiate Passiflora tarminiana from $P$. tripartita var. mollissima, it has never been mentioned as a distinct species in taxonomical studies. In a letter to Tarmín Campos, Linda de Escobar considered it to be a hybrid of $P$. tripartita var. mollissima, possibly with $P$. cumbalensis (Karst.) Harms. We have observed $P$. tarminiana almost everywhere in the Colombian highlands, as well as in the Andes of Venezuela, southern Ecuador, and Peru, with constant and distinct phenotypic traits. Plants grown from seeds show no segregation for these phenotypic traits, which would not be the case of a hybrid. Recent morphological and isozyme studies have confirmed that $P$. tarminiana is distinct from other common species of subge nus Tacsonia, such as $P$. tripartita var. mollissima, $P$. mixta L.f, $P$. cumbalensis, and P. pinnatistipula Cav. (Segura et al., 1998, Villacis et al., 1998). Passiflora tarminiana Coppens \& Barney sp. nov. 
TYPE: Colombia, Department Valle del Cauca. Tenerife (municipio El Cerrito), under cultivation, 2200-- $2600 \mathrm{~m}, 3^{\circ} 43.189^{\prime} \mathrm{N}, 76^{\circ} 04.482 \mathrm{~W}, 8$ March 1999. Coppens IPGRI-AM 72 (holotype, COL; isotypes, AAU, AK, BISH, CUZ, GOET, HUA, IPGRI, K, MEXU, MO, MYF, QCA, QCNE, TEX, US, VALLE, VEN). Figure 1--3. Table 1.

Haec species a $P$. tripartita var. mollissima (Kunth) Holm-Nielsen \& P. Jørgensen sepalos et petalos longioribus et perpendicularis o reflexos; nectarium mayoribus; stipulis menoribus et acicularibus.

Liana, stem cylindrical, bark fibrous ; internodes 6--12 cm long; indument canescent, soft to the touch. Stipules auricular and aristate $4--7 \times 2(--3) \mathrm{mm}(1--3 \mathrm{~mm}$ long without the aristate apex), arista 3--4 mm long, early deciduous. Petioles $1.5--4 \mathrm{~cm}$, slightly caniculate adaxially, canescent-ferruginously pubescent, 1--4 pairs of adaxial glands. Leaves trilobed (7--) 16 (--29) cm wide; lobes ovate, acuminate; margin serrate, central lobe (5.5--) $11(--16) \times(2.5--) 5(--8) \mathrm{cm}$, lateral lobes $(5--) 9(--16) \times(2.3--) 4(--$ 7) $\mathrm{cm}$; lamina moderately lustrous above, scarcely pubescent, trichomes short, although some very long; undersurface canescent-pubescent, the trichomes ferrugineous along the nerves. Flowers axillary, solitary, pendent; peduncles $3--10 \mathrm{~cm}$, canescent-pubescent; bracts 3--5 $\times 2--3 \mathrm{~cm}$, united halfway, margins entire, ovate, acuminate, nerves yellowish, reticulate venation visible, located $1 \mathrm{~cm}$ from hypanthium base; hypanthium (floral tube) 6--8 $\times$ 0.7--1 cm, light green outside, whitish inside; nectar chamber semiglobose, 1.4--2 cm wide; operculum reflexed, margin recurved; annulus present; sepals and petals bright pink to light pink, generally $64 \mathrm{D}$ or $75 \mathrm{~A}$ in the RHS Kew color chart, perpendicular to the hypanthium or reflexed; sepals $4.5--6 \times 1.2--2.5 \mathrm{~cm}$, oblong, aristate subterminally, awn 3--4 mm; petals 3--6 mm shorter than the sepals; corona tuberculate, white with 
purple base; androgynophore 7-- $10 \mathrm{~cm}$, white; free filaments $2 \mathrm{~cm}$, white; anthers yellow; ovary fusiform, green, pubescent; styles white, stigmas green. Fruit 10--14 × 3.5--4.5 cm, fusiform; young fruits canescent, the pericarp dark green with white dots except along the carpel seams, the dried styles persistent; during maturation dots disappearing and fruit turning yellow to orange yellow. Seeds asymmetrical, reddish brown when dry, reticulate, acute, cordate; arils orange, sweet, and aromatic.

Figure 2 shows the sites where $P$. tarminiana has been observed or collected in the Andes. Table 1 presents a comparison of $P$. tarminiana against two other common species of the subgenus Tacsonia. The most typical traits of $P$. tarminiana are the absent or reduced pubescence on the upper side of the leaves, the minute stipules that are almost always deciduous, the flower with a greater sepal/calyx tube ratio, as compared to other common species as $P$. tripartita or $P$. mixta. It is further characterized by reflexed light pink petals, a nectar chamber that is much wider than the calyx tube, a fusiform fruit, with small whitish dots which are evenly distributed on the pericarp before maturity, except on the aristas between the carpels. In comparison, the other widely cultivated banana passion fruit, $P$. tripartita var. mollissima, shows a marked pubescence on both leaf sides, permanent and larger stipules, a bell-shaped corolla, a longer calyx tube and shorter sepals and petals. The fruit of the cultigen P. tripartita var. mollissima is oblong with round extremities, and uniformly green before maturity (however P. tripartita var. tripartita may also show the whitish dots on the immature fruit). When both cultigens can be compared in the same orchard, $P$. tripartita var. mollissima shows much darker foliage, magenta flowers, and pale yellow mature fruits. The fruits of $P$. tarminiana are of a deeper yellow to orange color, and their pulp is less aromatic and tart. These differences 
in shape and color make them easy to recognize for the consumer and it is surprising that this species has escaped detection by botanists for so long.

According to the botanical keys of Colombia and Ecuador, P. tarminiana would key out to species with broadly ovate-reniform or auriculate and denticulate stipules and pendent peduncles, near $P$. cumbalensis and $P$. tripartita (P. mollissima in Escobar, 1988). Our new species can easily be distinguished from these species by the size and permanence of the stipules and the widening of the nectar chamber. Leaf pubescence is not useful to discriminate $P$. tarminiana, as both $P$. cumbalensis and $P$. tripartita, show variation for this trait. In Colombia, because P. tarminensis had not been described before as a distinct species, and because it is sometimes named "curuba quiteña" or "curuba ecuatoriana," some researchers confused it with P. tripartita var. tripartita, from Ecuador. This confusion is sometimes found in the "gray literature" (research reports and student theses, e.g. Sañudo and Jurado, 1990).

Confusion with $P$. tripartita var. mollissima or with supposed hybrids is frequent in the horticultural, weed science, and fruit culture literature. Pictures of flowers and fruits of $P$. tarminiana are frequently presented as those of $P$. tripartita var. mollissima (e.g., Vanderplank, 1996; Ulmer \& Ulmer, 1997; Wagner et al., 1999).

As commonly observed in the subgenus Tacsonia, $P$. tarminiana hybridizes easily with other species of the subgenus. The hybrids with P. mollissima and P. mixta are fertile and show intermediate phenotypes (pers. obs.). Hybrid seeds have also been obtained with P. cumbalensis (J.F. Restrepo, pers. com.).

Passiflora tarminiana is adapted to a wide range of elevations as compared to other species of subgenus Tacsonia growing at tropical latitudes. It may be cultivated 
from about $2000 \mathrm{~m}$ up to more than $3000 \mathrm{~m}$. In comparison, $P$. tripartita var. mollissima is not well adapted under $2400 \mathrm{~m}$. As in P. tripartita var. mollissima, the fruits of $P$. tarminiana grow larger at higher elevations. Passiflora tarminiana seems to be more resistant to fungi. We have never observed oidia on its leaves or a significant number of anthracnosis necrotic spots on its fruits. On the contrary, the pericarp of $P$. tripartita var. mollissima fruits is often affected by anthracnosis, which reduces its market value despite its superior taste. In Venezuela, $P$. tripatita var. mollissima has shown high susceptibility to fusariosis while P. tarminensis appeared tolerant or resistant (E. González, pers. com). The adaptative potential and rusticity of $P$. tarminensis have probably been key factors in its conquest of new habitats in New Zealand and Hawaii where it is reported as a noxious weed (La Rosa, 1984).

Villacis et al. (1998) studied morphological variation in an Ecuadorian field collection including the most common species of subgenus Tacsonia. The cluster analyses based on both quantitative and qualitative morphological data clearly separated accessions of $P$. tarminiana from the other species. Passiflora tarminiana appeared closer to $P$. tripartita var. mollissima and $P$. mixta than to $P$. cumbalensis and $P$. pinnatistipula. Similarly, using six isozyme systems, Segura et al. (1998) showed that P. tarminiana is clearly separated from $P$. tripartita var. mollissima, $P$. mixta, and P. cumbalensis, while P. tripartita var. mollissima and P. mixta could not be clearly distinguished from each other. In a study by Fajardo et al. (1998) with RAPD markers on a sample of 52 plants from seven subgenera and 14 species of passifloras, the eight plants of $P$. tripartita var. mollissima and five plants of the species here described (identified in the work as Passiflora sp. "india”) formed two different subclusters within the subgenus Tacsonia. 
Three of these five plants produced the same cpDNA RFLP pattern, distinct from that obtained on cpDNA from P. tripartita var. mollissima (Sánchez et al., 1999). Paratypes:

COLOMBIA. Cauca, Silvia, Barney IPGRI-AM-14 (IPGRI). Valle del Cauca, Barragán, $4^{\circ} 01.52^{\prime} \mathrm{N}, 75^{\circ} 53.54^{\prime} \mathrm{W}, 2900 \mathrm{~m}$, Coppens \& S. Segura IPGRI-AM-3 (IPGRI); Tenerife (municipio El Cerrito). under cultivation, 2200--2600 m, 3 $43.189^{\prime} \mathrm{N} 76^{\circ} 04.482 \mathrm{~W}$, same plants as type, Coppens \& Barney IPGRI-AM 2 (IPGRI, MO).

ECUADOR. Carchi, S of Tulcán, 0³0.52'N, 7754.05'W, 2690 m, Coppens \& Barney IPGRI-AM-13 (IPGRI). Chimborazo, Volcán Chimborazo, Barney IPGRI-AM-8 (IPGRI); Riobamba, Químiag, 2650 m, C. Tapia \& J. Velásquez CS-070 (INIAP). Loja, Santiago, $3^{\circ} 47.38^{\prime} \mathrm{S}, 7^{\circ} 17.38^{\prime} \mathrm{W}, 2450 \mathrm{~m}$, Coppens IPGRI-AM-10 (IPGRI); Saraguro, San Lucas, 2550 m, C. Tapia \& E. Morillo CTEM-040 (INIAP). Pichincha, cultivada, Parraoquia Calacali, Reserva Geobotánica Pululahua, 005'N, 78³0'W, Cerón \& Cerón 2740 (MO); 0²2'S, 78²5'W, 2650 m, Coppens \& Barney IPGRI-AM-12 (IPGRI); Unchibamba, S of Quito, $1^{\circ} 07.85^{\prime} \mathrm{S}, 78^{\circ} 35.32^{\prime} \mathrm{W}, 2610 \mathrm{~m}$, Coppens \& Barney IPGRIAM-11 (IPGRI); Rumiñahui, Iasa, 0²2'S, 78²5'W, 2650 m, N. Mazón \& B. Elizalde NMO-038 (INIAP). Tungurahua, Ambato, $1^{\circ} 22.02^{\prime} \mathrm{S}, 78^{\circ} 36.21^{\prime} \mathrm{W}, 2500 \mathrm{~m}$, Barney IPGRI-AM-9 (IPGRI); Baños, 2680 m, Coppens IPGRI-AM-4 (IPGRI). ETHIOPIA. Alemaya, cultivated, 2000 m, Jul 1967, Westphal \& Westphal-Stevels 494 (MO).

MEXICO. Distrito Federal, cultivated at El Rosario, 20 Aug 1936, MacDaniels 635 (BH). Michoacán, desv. a San José del Rincón carr. Angangeo--Villa Victoria, 2750 m, Soto \& Ramírez 1496 (MEXU, MO). Morelos, Mpio. Huitzilac, H. Hernández 16 
(MEXU); Mpio. Huitzilac, 1.2 km de la Carr. Federal Cuernavaca--México, D.F., rumbo a Zempoala, 2500 m, Luna C. 21 (MEXU, MO).

NEW ZEALAND. North Island, Mount Albert, 3654'S, 17444'E, Astridge (AK219103); Wellington, $41^{\circ} 17^{\prime} \mathrm{S}, 174^{\circ} 46^{\prime} \mathrm{E}$, Brownsey (AK-152731); Titirangi, 360ㅗ'S, $174^{\circ} 03^{\prime} \mathrm{E}$, Cameron (AK-221386); Grafton Gully, 36 $52^{\circ} \mathrm{S}, 174^{\circ} 46^{\prime} \mathrm{E}, 50 \mathrm{~m}$, Cameron (AK-221519); Waiheke Island, 36²99'S, 17507'E, 80 m, Cameron 7524 (AK-229217); Swanson, 36²5'S, 174³4'E, 100 m, Cameron 9458 (AK-236385); Paihia, 35¹7'S, 17406'E, Cumber (AK-116173); Mount Albert, 3654'S, 17444'E, Dingley (AK122719); Palmerston, $40^{\circ} 24^{\prime} \mathrm{S}, 175^{\circ} 33^{\prime} \mathrm{E}$, Esler (AK-173113); Mount Albert, 3654'S, 17444'E, Esler (AK-219104), Esler (AK-219109); Kerikeri, 35¹4'S, 17357'E, Esler (AK-219107); Wood Bay, 3657'S, 17440'E, Esler (AK-219108); between Nelson City and Whakapuaka, Healy 74/80 (MO); Waitemata, 36 $57^{\circ} \mathrm{S}, 174^{\circ} 35^{\prime} \mathrm{E}, 240 \mathrm{~m}$, Mackinder (AK-162676); Tutukaka and Matapouri, 3535'S, 174³1'E, Newfield (AK-212296);

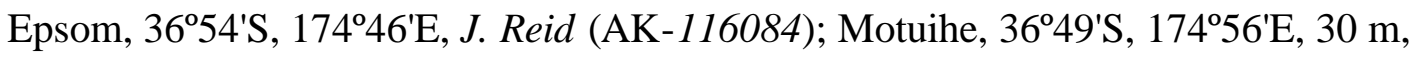
Sikes (AK-220536); Buller, Karamea, near Karamea, Sykes 10/85 (MO); Hokianga,

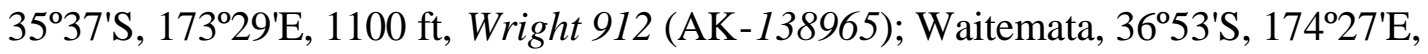
Wright 1657 (AK-140997); Mount Albert, 3654'S, 1744''E, B. Young (AK-114204), B. Young (AK-114205), B. Young (AK-116172), B. Young (AK-117558); Titirangi, 3605'S, $174^{\circ} 03^{\prime} \mathrm{E}$, B. Young (AK-116171), B. Young (AK-117563); Otahuhu, 3655'S, 17451'E,

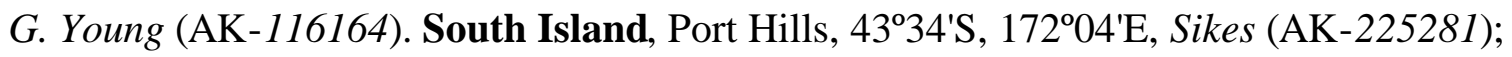
Punakaiki, 42007'S, 171²0'E, B. Young (AK-117584), B. Young (AK-221387). PANAMA. Chiriqui, carr. hacia la cima del Volcán Barú, Montenegro 1630 (MO). 
PAPUA NEW GUINEA. New Guinea Group, New Guinea, Eastern Highlands province, Mount Wilhelm near Iwam Pass, 549'S, 14507'E, 2800 m, Takeuchi 5898 (MO). PERU. Arequipa, Tuhuana; 15³9'06"S, 72²8'09"W, 2545 m, Ll. Rios, J. Medina \& L. López INIA-PRONARGEB 230 (INIA). Cajamarca, Barrio Santa Elena; 7¹7'51"S, 7851'56"W, 2730 m, Ll. Rios, J. Medina \& L. López INIA-PRONARGEB 170 (INIA); alrededores de Guzmango, Prov. Contumazá, 2600-2700 m, Sagástegui A. 122 (US). Huanuco, Chinchao, 972'72"S, 7609'68"W, 2650 m, Ll. Rios, J. Medina \& L. López. INIA-PRONARGEB 101 (INIA), 2480 m, Ll. Rios, J. Medina \& L. López INIAPRONARGEB 104 (INIA); Soldado Ucro, 980'90"S, 7608'00"W, 3200 m, Ll. Rios, J. Medina \& L. López INIA-PRONARGEB 113 (INIA); Conchamarca, 1003'99"S, 76²0'35", 2490 m, Ll. Rios, J. Medina \& L. López INIA-PRONARGEB 116 (INIA); La Libertad-Ting, 1001'56"S, 76²17'00"W, 2820 m, Ll. Rios, J. Medina \& L. López INIAPRONARGEB 118 (INIA); Quiulacocha, 1001'56", 76¹7'00"W, 2820 m, Ll. Rios, J. Medina \& L. López INIA-PRONARGEB 120 (INIA).

U.S.A. California, cultivated “in Southern California,” Jul-Aug 1915, Boughton 242 (US); Golden Gate Park, San Francisco, Aug 1907, Wight 1806 (MO). Hawaii, Hwy. 550 along Waimea Canyon, mile 14 near NASA tracking station, 3500 ft, Croat 44833 (MO); Kauai, rd. to Kumuwela Lookout, 2206'N, 159³9'W, 3400 ft, Crosby \& Anderson 1496 (DUKE); Hawaii, Muana Kea, Aug 1949, Degener et al. 20354 (MO); Kauai, near Kokee Ranger Station, Degener \& Degener 35181 (MO); Hawaii, Puna, Hawaii Volcano National Park, Degener \& Degener 35183 (MO); Kauai, Henrickson 4034 (NCU); Puna district, land of Olaa, 1200 m, D. Horbst (MYF-459); Kauai, Waimea District, Na PaliKona Forest Reserve, Makaha Valley, 870--950 m, Lorence 5221 (MO); Kauai, Pu'u 
Hinahina Lookout, Waimea Canyon, 640 m, Thorne \& Zupan 10153 (MO); Hawaii, Muana Kea road by Douglass Monument, 5000 ft, Trujillo s.n. (MO); Hawaii, North Kona, Puuwaawaa, 3000 ft, Jun 1948, Webster \& Wilbur 1853 (DUKE).

See also description of localities and ecology in Hawaii by La Rosa (1984).

VENEZUELA. Junín, Betania,Villa Paez, 7³1.70'N, 72²6'W, 2000 m, E. González \& Barney IPGRI-AM-5 (IPGRI); Mérida, Mucuruba, 809.46'N, 71²0'W, 2000 m, E. González \& Barney IPGRI-AM-6 (IPGRI). Táchira, Pueblo Hondo, 8¹5.19'N, 71'53.07'W, 2500 m, E. González \& Barney IPGRI-AM-7 (IPGRI).

ZIMBABWE. Distr. Inyanga, Inyanga Downs, naturalized in Kloof, near sawmill, Jan 1981, Geddes s.n. (MO).

Germplasm collections:

COLOMBIA. Boyacá, Nuevo Colón, $5^{\circ} 21.12^{\prime} \mathrm{N}, 73^{\circ} 27.70^{\prime} \mathrm{W}, 2450 \mathrm{~m}$, S. Segura \& L. López TEN 63 (CIRAD-FLHOR/IPGRI). Cauca, Inzá, Coppens TEN 83 (CIRADFLHOR/IPGRI). Cundinamarca, San Bernardo, 409.05', 74²3.50', 2010 m, S. Segura \& L. López TEN 50 (CIRAD-FLHOR/IPGRI). Putumayo, Santa Clara, Sibundoy, 2700 m, Coppens TEN 58 (CIRAD-FLHOR/IPGRI). Valle del Cauca, Barragán, $4^{\circ} 01.52^{\prime} \mathrm{N}$, 7553.54'W, 2900 m, Coppens \& S. Segura TEN 5 (CIRAD-FLHOR/IPGRI).

ECUADOR. Loja, Loja, Coppens TEN 21 (CIRAD-FLHOR/IPGRI).

Field observations: 
COLOMBIA. Caldas, Manizales, $5^{\circ} 02^{\prime} \mathrm{N}, 75^{\circ} 27^{\prime} \mathrm{W}$, observ. by Coppens. Nariño, La Cocha, $1^{\circ} 09.11^{\prime} \mathrm{N}, 77^{\circ} 09.25^{\prime} \mathrm{W}$, observ. by Coppens; San Francisco, $1^{\circ} 09.73 \mathrm{~N}, 77^{\circ} 0^{\prime} \mathrm{W}$, 2140 m, observ. by Coppens.

FRANCE. La Réunion, recently introduced at elevations over 700 m, observation and documentation by C. Lavigne (in letter with photography).

\section{Acknowledgments}

The abbreviations INIA, INIAP, IPGRI, and CIRAD-FLHOR/IPGRI used in the citations of specimens correspond to the following institutions: INIA-Instituto Nacional de Investigación Agraria, Perú, Casilla 2791, Lima 1, Peru; INIAP_-Instituto Nacional de Investigaciones Agropecuarias, Ecuador, C.P. 17-01-340, Quito, Ecuador; IPGRIInternational Plant Genetic Resources Institute, IPGRI-AM, oficina para las Americas c/o CIAT, A.A. 6713, Cali, Colombia; CIRAD-FLHOR/IPGRI-field germplasm collections in Tenerife, Colombia, maintained by CIRAD-FLHOR/IPGRI Project for Neotropical Fruits, address above. This research has been partly funded by Colciencias through the project 1203-12-097-98: "conservación y utilización de los recursos genéticos de pasifloras.” The authors thank Professor S. Tillet (MYF), Ing. Elide Gonzalez (FONAIAP), Dr. Jaime Estrella and Ing. Nelson Mazón (INIAP), Ing. Jorge Vega (Universidad Técnica de Ambato), Ing. Llermé Ríos (INIA), and Dr. C. Lavigne (CIRAD-FLHOR) for helping in collecting or by providing herbarium material, Luigi Guarino (IPGRI) for mapping the distribution of the species, and Sophie Primot and Vincent Rioux for gathering morphological data for Table 1.

\section{References}


A. A. A. (Asistencia Agroemprearial Agribusiness). 1992. Manual técnico del taxo. Editorial Ecuador, Quito, 31 pp.

Campos, T. C. 1992. El cultivo de la curuba (Passiflora mollissima (H.B.K.) Bailey) en Colombia. Acta Hort. 310: 215--229.

Escobar, L. K. 1980. Interrelationships of the edible species of Passiflora centering around Passiflora mollissima (H.B.K.) Bailey subgenus Tacsonia. Doctoral Thesis University of Texas, Austin, USA.

Escobar, L. K. 1988. Monografía No. 10. Passifloraceae. Passiflora. Subgéneros:

Tacsonia, Rathea, Manicata \& Distephana. Universidad Nacional de Colombia, Bogotá, D.E., Colombia, 138 pp.

Fajardo, D., Angel, F., Grum, M., Tohme, J., Lobo, M., Roca, W. M. and Sánchez, I. 1998. Genetic variation analysis of the genus Passiflora L. using RAPD markers. Euphytica 101: 341--347.

Holm-Nielsen, L. B., Jørgensen, P. M. and Lawesson, J. E. 1988. 126. Passifloraceae. No. 31. Flora of Ecuador. Berlings, Arlöv, Copenhagen, Denmark, 129 pp.

Jaramillo, A. 1957. Primeros resultados de un ensayo sobre el cultivo de la curuba (Passiflora spp.). Agricultura tropical 13 (5): 301--308.

La Rosa, A. M. 1984. The biology and ecology of Passiflora mollissima in Hawaii. Cooperative National Park Resources Studies Unit. Technical Report 50, 147 pp.

Pérez Arbeláez, E. 1978. Plantas utiles de Colombia. Litografía Arco, Bogotá, Colombia, $831 \mathrm{pp}$.

Sánchez, I., Angel, F., Grum, M., Duque, M.C., Lobo, M., Tohme, J. and Roca, W. 1999. Variability of chloroplast DNA in the genus Passiflora L. Euphytica, 106: 15--26. 
Sañudo, B. and Jurado, D. 1990. Búsqueda de fuentes de resistencia a enfermedades fungosas de la curuba en Nariño. Ascolfi informa, 16(1) : 3.

Segura, S. D., Coppens d'Eeckenbrugge and G., Ollitrault, P. 1998. Isozyme variation in five species of Passiflora subgenus Tacsonia and Passiflora manicata. Proc. Interamerican Soc. Trop. Hort. 42:260--266.

Ulmer, T. and Ulmer, B. 1997. Passionsblumen: eine faszinierende Gattung. pp 5--384. Laupenmúhlen Druck, Witten.

Vanderplank, J. 1996. Passion flowers. Cassel, London, 224pp.

Villacis, L. A., Vega, J., Grum, M. and Coppens d'Eeckenbrugge, G. 1998. Morphological characterization of Andean passifloras (Passiflora spp.) from Ecuador. Plant Gen. Res. Newsl. 115 : 51--55.

Wagner, W. L, Herbst, D. A., and Sohmer, S. H. 1999. Manual of the Flowering Plants of Hawai'i. Rev. ed., vol. 2: 989--1919. University of Hawai'i Press/ Bishop Museum Press.

Young, B. R. 1970. Identification of passionflowers in New Zealand (Dicotyledones: Passifloraceae). Rec. Auckland Inst. Mus. 7: 143--169. 
Table 1. Comparison of three common or cultivated species of Passiflora subgenus Tacsonia

\begin{tabular}{|c|c|c|c|}
\hline & P. tarminiana & P. tripartita & P. mixta \\
\hline Stem & terete & terete & subangular \\
\hline Leaf pubescence & $\begin{array}{l}\text { absent on upper } \\
\text { surface, moderate } \\
\text { on lower surface }\end{array}$ & $\begin{array}{l}\text { var. mollissima: } \\
\text { dense on both sides; } \\
\text { other varieties: } \\
\text { variable, often } \\
\text { glabrous at least on } \\
\text { upper surface }\end{array}$ & $\begin{array}{l}\text { most often glabrous, } \\
\text { occasionally } \\
\text { pubescent }\end{array}$ \\
\hline Stipules & $\begin{array}{l}\text { small (4--7 mm } \\
\text { long, 2--6 mm } \\
\text { wide), subreniform, } \\
\text { denticulate or } \\
\text { serrulate, deciduous }\end{array}$ & $\begin{array}{l}\text { medium (6--12 mm } \\
\text { long, } 13--19 \mathrm{~mm} \\
\text { wide), reniform, } \\
\text { serrulate to serrate, } \\
\text { permanent }\end{array}$ & $\begin{array}{l}\text { medium to large (6-- } \\
20 \mathrm{~mm} \text { long, 12--30 } \\
\text { mm wide), } \\
\text { reniform, dentate or } \\
\text { serrate, permanent }\end{array}$ \\
\hline Peduncle & $\begin{array}{l}\text { slender, variable in } \\
\text { length, flower } \\
\text { pendent }\end{array}$ & $\begin{array}{l}\text { slender, short, } \\
\text { flower pendent }\end{array}$ & $\begin{array}{l}\text { stout, variable in } \\
\text { length, flower half- } \\
\text { pendent to erect }\end{array}$ \\
\hline Bracts & $\begin{array}{l}\text { united } 1 / 2 \text { their } \\
\text { length or more }\end{array}$ & $\begin{array}{l}\text { united } 1 / 2 \text { their } \\
\text { length or more }\end{array}$ & $\begin{array}{l}\text { united on } 3 / 4 \text { of their } \\
\text { length or more }\end{array}$ \\
\hline Flowers & $\begin{array}{l}\text { light pink (Kew } \\
\text { color chart: red- }\end{array}$ & $\begin{array}{l}\text { pink to magenta } \\
\text { (Kew color chart: }\end{array}$ & $\begin{array}{l}\text { light pink to bright } \\
\text { red (Kew color }\end{array}$ \\
\hline
\end{tabular}




\begin{tabular}{|c|c|c|c|}
\hline & $\begin{array}{l}\text { purple group, 57D, } \\
66 \mathrm{D}, 74 \mathrm{D}, 75 \mathrm{~A} / \mathrm{B} / \mathrm{C} \text {, } \\
\text { rarely white), } \\
\text { corolla reflex, calyx } \\
\text { tube/corolla length } \\
\text { ratio around } 1.3-1.6 \text {, } \\
\text { nectary chamber } \\
\text { appreciably wider } \\
\text { than the calyx tube }\end{array}$ & $\begin{array}{l}\text { red-purple group, } \\
\text { 57C/D, 62A, 65A, } \\
\text { 66C-D, 68B, 70D } \\
\text { for var. mollissima, } \\
\text { 53B for var. } \\
\text { azuayensis, 58B for } \\
\text { var. tripartita), } \\
\text { corolla } \\
\text { campanulate, calyx } \\
\text { tube/corolla length } \\
\text { ratio around 2.4-3.2, } \\
\text { nectary chamber } \\
\text { slightly wider than } \\
\text { the calyx tube }\end{array}$ & $\begin{array}{l}\text { chart: most often } \\
\text { red/orange-red } \\
\text { group, 39A, 42A, } \\
\text { 50A, 51B, 52B, } \\
\text { 54A, 54B, 55A, also } \\
\text { red-purple group in } \\
\text { southern Colombia } \\
\text { and Ecuador, } \\
\text { 63C/D, 75B), } \\
\text { corolla } \\
\text { campanulate, calyx } \\
\text { tube/corolla length } \\
\text { slightly wider than } \\
\text { nectio around 1.6-2.6, } \\
\text { nealyx tube }\end{array}$ \\
\hline Fruits & $\begin{array}{l}\text { pericarp yellow, } \\
\text { sometime orange- } \\
\text { tinged, arils orange } \\
\text { and succulent }\end{array}$ & $\begin{array}{l}\text { pericarp pale yellow } \\
\text { (var. mollissima) to } \\
\text { yellow (var. } \\
\text { tripartita), arils } \\
\text { orange and } \\
\text { succulent }\end{array}$ & $\begin{array}{l}\text { pericarp often green } \\
\text { at maturity, } \\
\text { sometimes turning } \\
\text { yellow, arils grey to } \\
\text { orange, scant }\end{array}$ \\
\hline
\end{tabular}


Figure 1. Passiflora tarminiana Coppens \& Barry. -- A. Habit with bud and flower at anthesis. --B. Fruit. --C. Longitudinal section of hypanthium and floral tube showing nectary chamber, operculum, and reduced corona. --D. Node showing stipules. --E. Seed.

Figure 2. Distribution of Passiflora tarminina Coppens \& Barney in northwestern South America, circles.

Figure 3. Color photos of the plant that provided the type material. 